\title{
Free Logic and the Quantified Argument Calculus
}

\section{Pavlovic, Edi}

de Gruyter

2019

Pavlovic , E \& Gratzl , N 2019 , Free Logic and the Quantified Argument Calculus . in G M Mras , P Weingartner \& B Ritter (eds), Philosophy of Logic and Mathematics : Proceedings of the 41st International Ludwig Wittgenstein Symposium . Publications of the Austrian pÿLudwig Wittgenstein Society New Series , no. 27 , de Gruyter , pp. 105-116 , International Ludwig Wittgenstein Symposium , Kirchberg am Wechsel , Austria , 05/08/2018 . https://doi.org/10.1515/978311065

http://hdl.handle.net/10138/320976

https://doi.org/10.1515/9783110657883-007

unspecified

acceptedVersion

Downloaded from Helda, University of Helsinki institutional repository.

This is an electronic reprint of the original article.

This reprint may differ from the original in pagination and typographic detail.

Please cite the original version. 


\title{
Free Logic and the Quantified Argument Calculus
}

\author{
Edi Pavlović*1 and Norbert Gratzl ${ }^{2}$ \\ ${ }^{1}$ University of Helsinki, Finland, edi.pavlovic@helsinki.fi \\ ${ }^{2}$ Ludwig Maximilian University of Munich, Germany, N.Gratzl@lmu.de
}

\begin{abstract}
The Quantified Argument Calculus (or Quarc for short) is a novel and peculiar system of quantified logic, particularly in its treatment of nonemptiness of unary predicates, as in Quarc unary predicates are never empty, and singular terms denote. Moreover, and as a consequence of this, the universally quantified formulas entail their corresponding particular ones, similar to existential import. But at the same time, Quarc eschews talk of existence entirely by having a particular quantifier instead of an existential one. To bring it back into consideration, we explicitly introduce the existence predicate, and modify the rules to make the existence assumption obvious. This, along with some modifications, leads to a version of negative free logic. A question that arises at this point, given that we are interested in free logic, is what happens when we remove the existence assumption on singular terms; here we can quite naturally choose the negative free logic framework as well. In this paper we shall therefore investigate interrelations between Quarc and free logic (especially with its negative variant), and approach these interrelations with proof-theoretic methods.
\end{abstract}

\section{Introduction}

Classical Logic (CL) is the most well-known approach to formal reasoning; it has its own quirks and features. Recently, a new alternative to CL has been developed. One of the reasons for developing a new alternative to CL is to provide for formal reasoning and formalization processes that are (arguably) closer to natural language. This system (or rather family of system) is called Quantified argument calculus (Quarc for short), c.f. e.g. [4], [12], [15].

In the 1960ies CL has been investigated with respect to its specific existence assumptions and the outcome has been (again a family of) free logics, where free logic is short for first order logic free of existence assumptions. Existence

*This work was partially supported by the Academy of Finland, research project no. 1308664 
assumptions vary but the central claims are: (1) the domain of an interpretation is not empty (this is respected in the CL theorem $\exists x(x=t)$, (2) every name denotes exactly one object in the domain, and (3) the quantifiers have existential import (expressed by $\forall x E ! x$ ).

Neither CL nor Free Logic, however, have existence assumptions on unary predicates. This is not so in Quarc - in Quarc unary predicates are never empty, and singular terms denote. But at the same time, Quarc eschews talk of existence entirely by having a particular quantifier instead of an existential one. A particular quantifier tells us that there is an instance of the unary predicate, so in this sense the said predicate is not empty. Furthermore, of this instance (at least) something can be truthfully predicated. So, in this sense it expresses that "there are" things, but is stops short of identifying this construction with the existence statement about it, and therefore remains agnostic on the claim of existence (it's possible we could say true things about non-existents, such as, for example, that there are some). To bring it back into the discussion, we explicitly introduce the existence predicate, modify the rules to make the existence assumption obvious, and introduce the rule for the new predicate. This leads to a version of negative free logic, and we investigate the versions both with and without identity.

In this paper we shall therefore investigate interrelations between Quarc and free logic (especially with its negative variant). Furthermore, this paper approaches these interrelations with proof-theoretic methods and results of [15]. In it the authors claim that the rules of quantification in (the family of logics) Quarc resemble those of free logic. The results of this paper substantiate that claim.

\subsection{Quarc}

Quarc is a system of quantified logic which does away with variables and unrestricted predicates, but nonetheless achieves results similar to the Predicate Calculus by employing quantifiers, applied directly to unary predicates, which then appear as arguments of other predicates (hence the name Quantified Argument Calculus), along with operators that attach to predicates and subsequently modify the mode of predication, as well as anaphors. It is in these respects, as mentioned previously, closer to natural language.

Let us note that the quantifiers in Quarc do have particular import, a fact that is expressed semantically by the condition of non-emptiness of (unary) predicates. This is in contrast to first-order predicate logic, where, as it is well known, (unary) predicates can be empty. For the purpose of investigating logics free of existence assumptions, we will in the proceeding also eliminate this condition ${ }^{1}$, and focus on the resulting systems, labelled Quarc $_{B}$ for version

\footnotetext{
1 [13] investigates a three-valued version of Quarc that also omits this assumption. Here, however, we will remain within the confines of a two-valued system.
} 
without identity, and $\mathrm{Quarc}_{2}$ for version with it, as well as their respective sequent-calculus representations, LK-Quarc $B$ and LK-Quarc 2 .

\section{Free Logic}

Let us start with admittedly very broad, but nonetheless instructive, explanation of what free logics are:

A free logic is a formal system of quantification theory, with or without identity, which allows for some singular terms in some circumstances to be thought of as denoting no existing object, and in which quantifiers are invariably thought of as having existential import. [2, 148-149]

In this quote one might glimpse a connection between Quarc and free logics - the quantifiers having existential import. Of course, given that Quarc doesn't talk of existence, the connection will have to be refined in the proceeding, but this will serve as an initial point of contact - both Quarc and free logic challenge the standard commitments to existence. A more formal way to characterize (negative, as it among the many free logics will be the sole focus of this paper) free logic would be to describe it via axioms:

1. $\forall x(A \rightarrow B) \rightarrow(\forall x A \rightarrow \forall x B)$

2. $A \rightarrow \forall x A$, if $x$ is not free in $A$

3. $\forall x A \rightarrow(E ! t \rightarrow A t)$

4. $\forall x E ! x$

5. $R\left(t_{1}, \ldots, t_{n}\right) \rightarrow\left(E ! t_{1} \wedge \ldots \wedge E ! t_{n}\right)$

6. $\forall x(x=x)$

7. $s=t \rightarrow(A s \rightarrow A t)$

On the other hand, in a sequent calculus the rules for quantifiers in free logic can be formulated as follows, following [2] and slightly simplified:

$$
\begin{array}{lr}
\frac{A[t / x], \Gamma \Rightarrow \Delta \quad \Gamma \Rightarrow \Delta, E ! t}{\forall x A, \Gamma \Rightarrow \Delta} \mathrm{L} \forall & \frac{E ! t, \Gamma \Rightarrow \Delta, A[t / x]}{\Gamma \Rightarrow \Delta, \forall x A} \mathrm{R}^{*} \\
\frac{E ! t, A[t / x], \Gamma \Rightarrow \Delta}{\exists x A, \Gamma \Rightarrow \Delta} \mathrm{L} \exists^{*} & \frac{\Gamma \Rightarrow \Delta, E ! t \quad \Gamma \Rightarrow \Delta, A[t / x]}{\Gamma \Rightarrow \Delta, \exists x A} \mathrm{R} \exists
\end{array}
$$

${ }^{*}-t$ does not occur below the inference line. 
One can see that in all of these cases an extra requirement has been added - that of $E$ !t. In the following section we will employ the same principle to transform Quarc into its free version.

\section{$3 \quad$ Free Quarc}

To produce the free versions of the systems LK-Quarc ${ }_{B}$ and LK-Quarc ${ }_{2}$ we add the new rule for the existence predicate $E$ !, replace the rules for the quantifiers, and also replace one of the identity rules in the latter of the two systems. In the interest of brevity, the full systems will not be laid out here, but the reader can find both those, and a thorough discussion of their metatheoretical properties, in [15].

\subsection{The base system $-\mathbf{F Q}_{B}$}

To transform the system LK-Quarc ${ }_{B}$ (which does not contain identity) into a system of free logic $\mathrm{FQ}_{B}$, we modify the quantifier rules by an explicit condition on the existence of the singular term, in the same vein as above:

$$
\begin{aligned}
& \frac{A[a / \forall M], \Gamma \Rightarrow \Delta \quad \Gamma \Rightarrow \Delta, a M \quad \Gamma \Rightarrow \Delta, a E !}{A[\forall M], \Gamma \Rightarrow \Delta} \mathrm{L} \forall \quad \frac{a M, a E !, \Gamma \Rightarrow \Delta, A[a / \forall M]}{\Gamma \Rightarrow \Delta, A[\forall M]} \mathrm{R} \forall^{*} \\
& \frac{a M, a E !, A[a / \exists M], \Gamma \Rightarrow \Delta}{A[\exists M], \Gamma \Rightarrow \Delta} \mathrm{L} \exists * \quad \frac{\Gamma \Rightarrow \Delta, a M \quad \Gamma \Rightarrow \Delta, a E ! \quad \Gamma \Rightarrow \Delta, A[a / \exists M]}{\Gamma \Rightarrow \Delta, A[\exists M]} \mathrm{R} \exists
\end{aligned}
$$

${ }^{*}-a$ does not occur below the inference line.

In addition to the rules for quantifiers, we also supply the rule for the (negative free logic) existence predicate:

$$
\frac{a E !, A[a], \Gamma \Rightarrow \Delta}{A[a], \Gamma \Rightarrow \Delta} \mathrm{N} E !^{*}
$$

* $-A$ is basic $^{2}$.

With these in place, we can demonstrate the following axioms of negative free logic. Given that the system we were expanding did not contain identity, the resulting system will likewise not contain it.

\footnotetext{
${ }^{2}$ In the terminology of Quarc, a basic formula corresponds to an atomic formula of PC.
} 
Theorem 1 All of the following axioms of negative free logic are derivable in $\mathrm{FQ}_{B}$ :

1. $\left(\forall M_{\alpha} A \rightarrow \alpha B\right) \rightarrow(\forall M A \rightarrow \forall M B)^{3}$

2. $A \rightarrow(\forall M M \wedge B)$

3. $\forall M A \rightarrow((a E ! \wedge a M) \rightarrow a A)$

4. $\forall M E$ !

5. $A\left[a_{1}, \ldots, a_{n}\right] \rightarrow\left(a_{1} E ! \wedge \ldots \wedge a_{n} E !\right)$

Note that while these axioms characterize negative free logic, only axioms 3-5 are specific to it (i.e. 1-2 are likewise derivable in LK-Quarc $B$ ).

\subsection{Metatheoretic properties of $\mathbf{F Q}_{B}$}

We now turn towards establishing some metatheoretic properties of $\mathrm{FQ}_{B}$, first and foremost being the Cut elimination theorem. As everywhere in this paper, the proof is omitted, but it is a straightforward adaptation of the one found in [15].

Theorem 2 The Cut elimination property holds of $\mathrm{FQ}_{B}$. Namely, any sequent derivable in $\mathrm{FQ}_{B}$ is derivable without using the Cut rule.

To consider some consequences of this theorem, we first define the Subformula property, specifically its weak version (which will suffice to establish the results required in this paper).

Definition 3 (Weak subformula property) A sequent calculus system possesses the Weak subformula property just in case any formula occurring anywhere in a derivation of an endsequent is either a subformula of some formula occurring in that endsequent, or a basic formula.

It follows straightforwardly from Theorem 2 that

Corollary $4 \mathrm{FQ}_{B}$ possesses the Weak subformula property.

From this Corollary we can further demonstrate that

Corollary $5 \mathrm{FQ}_{B}$ is consistent.

This Corollary represents a desirable property of a logical system, but will not be of particular note going forward. Quite the opposite holds of the following one, however.

\footnotetext{
${ }^{3}$ This simplified formulation assumes the formula $\forall M B$ is governed by the Quantified Argument $\forall M$. Otherwise, a more involved form, namely $\forall M_{\alpha} M \wedge \alpha B$, must be used. Similar for the next axiom.
} 
Corollary $6 \mathrm{FQ}_{B}$ is a conservative extension of LK-Quarc ${ }_{B}$. Namely, any derivation $\Gamma \Rightarrow \Delta$ derivable in $\mathrm{FQ}_{B}$ and such that $\Gamma, \Delta$ do not contain $E$ ! is likewise derivable in LK-Quarc ${ }_{B}$.

We will discuss these properties of $\mathrm{FQ}_{B}$ at some length in Section 4, but for the moment we will examine adding the identity predicate to the system at hand.

\subsection{Adding identity $-\mathrm{FQ}_{2}$}

We add the rule for identity into the mix. Given the close connection of identity and the existence predicate in negative free logic, it should come as no surprise that the rules for the two look the same. We add this rule (it replaces the rule $={ }_{1}$ of LK-Quarc 2 ) and the rule $=_{2}$ to $\mathrm{FQ}_{B}$ to produce the system $\mathrm{FQ}_{2}$.

$$
\frac{a=a, A[a], \Gamma \Rightarrow \Delta}{A[a], \Gamma \Rightarrow \Delta} \mathrm{N}={ }^{*}
$$

${ }^{*}-A$ is basic.

With the addition of the identity rules, we can now derive the remaining axioms:

Theorem 7 In addition to those axioms mentioned in Theorem 1, the following are derivable in $\mathrm{FQ}_{2}$ :

6. $\forall M_{\alpha}=\alpha$, for any $M$

7. $s=a \rightarrow(s A \rightarrow a A)$

In addition to Theorem 7, several other results characteristic of a negative free logic are now derivable, namely

Theorem 8 Equivalence of existence and self-identity, $a E$ ! $\leftrightarrow a=a$, and indiscernibility of non-existents, $(a \neg E ! \wedge b \neg E !) \rightarrow(A[a] \rightarrow A[b / a])$, are both derivable in $\mathrm{FQ}_{2}$.

\subsection{Metatheoretical properties of $\mathrm{FQ}_{2}$}

Not much needs to be added here, as the results of this section closely resemble their counterparts in Section 3.2. It is straightforward to show that

Theorem 9 Cut elimination property holds for $\mathrm{FQ}_{2}$.

And from this it again follows that

Corollary $10 \mathrm{FQ}_{2}$ possesses the Weak subformula property. 
And again,

Corollary $11 \mathrm{FQ}_{2}$ is consistent.

An interesting consequence of Corollary 10 is

Corollary $12 a=a$ is not derivable in $\mathrm{FQ}_{2}$.

This corollary is of course, combined with Axiom $6(\forall x(x=x))$, characteristic of the way negative free logic treats the truth of self-identity sentences.

\section{Comparing Quarc and Free Quarc}

We have already seen that $\mathrm{FQ}_{B}$ is a conservative extension of LK-Quarc ${ }_{B}$. Now we move on to compare their respective versions containing identity, $\mathrm{FQ}_{2}$ and LK-Quarc 2 . Given the equivalence of existence and self-identity in $\mathrm{FQ}_{2}$, (Theorem 8), it will suffice that we observe the $E$ !-free segment of $\mathrm{FQ}_{2}, \mathrm{FQ}_{2}^{*}$. The result we obtain in this case is that

Theorem $13 \mathrm{FQ}_{2}^{*}$ is a proper subset of LK-Quarc, $\mathrm{FQ}_{2}^{*} \subset \mathrm{LK}$-Quarc 2 . Namely, every rule of $\mathrm{FQ}_{2}^{*}$ is admissible in LK-Quarc 2 , but (Corollary 12), $a=a$ is not derivable in $\mathrm{FQ}_{2}^{*}$.

This result should not come as a great surprise - in general, free logic is a restriction on classical logic. In this particular case, if we compare the differing identity rules in LK-Quarc 2 and $\mathrm{FQ}_{2}$, respectively:

$$
\frac{a=a, \Gamma \Rightarrow \Delta}{\Gamma \Rightarrow \Delta}={ }_{1} \quad \frac{a=a, A[a], \Gamma^{\prime} \Rightarrow \Delta}{A[a], \Gamma^{\prime} \Rightarrow \Delta} \mathrm{N}={ }^{*}
$$

* $-A$ is basic.

We can see that the latter is really just a special case of the former - specifically, when $\Gamma$ stands for the list of formulas $A[a], \Gamma^{\prime}$. By placing a limitation on the rules of the system, we likewise limit the output of the said system.

But now it should strike us as most peculiar that the same situation did not occur in the case of LK-Quarc ${ }_{B}$ and $\mathrm{FQ}_{B}$. Much like the rules for identity above, the quantifier rules of $\mathrm{FQ}_{B}$ impose a limitation on the corresponding rules of LK-Quarc ${ }_{B}$. And yet, we experience no loss of power (Corollary 6) - in fact, the only change has to do with the change in vocabulary that results from adding the existence predicate $E$ !. This anomaly bears closer scrutiny. 


\subsection{Comparison of $\mathrm{FQ}_{B}$ and LK-Quarc ${ }_{B}$}

As mentioned when we first introduced free version of Quarc, we produce it by means of an additional restriction on the rules. This, however, as we have just seen, does not result in a loss of expressive power. Normally, the most notable formula that becomes underivable in free logic is unrestricted specification, $\forall x A x \rightarrow A t$. Instead, we have the weaker, restricted specification (Axiom $3)$. The corresponding version of unrestricted specification in Quarc would be:

Definition 14 (Unrestricted specification) $\forall M A \rightarrow(a M \rightarrow a A)$

This formula can be obtained even in the free version LK-Quarc ${ }_{B}$ :

Theorem $15 \forall M A \rightarrow(a M \rightarrow a A)$ is derivable in $\mathrm{FQ}_{B}$.

The formula follows from Axiom 3 (restricted specification, $\forall M A \rightarrow((a E ! \wedge$ $a M) \rightarrow a A)$ ) and an instance of Axiom $5, a M \rightarrow a E !$. The latter is what explains this anomaly - in negative free logic, there is a close connection between the truth of atomic sentences and existence, expressed by Axiom 5 (and the absence of the formula $a=a$ ). But in $\mathrm{FQ}_{B}$, we only added the condition, in the appropriate place, depending on the rule, that $a E$ ! (thus allowing for the derivation of some formulas containing the new predicate), but the $a M$ requirement was already present in the non-free rule, i.e. the quantification was already restricted, and precisely in a manner that precludes the derivation of that formula which the free logic avoids. This demonstrates the point raised in [15],

Observation 16 The quantification rules of Quarc, even on the non-free version, have a "free flavor", or a structural resemblance to those of free logic.

This point is further strengthened in the following section, when we discuss free logic in relation to quantified arguments.

\section{$5 \quad$ Empty Predicates}

Given that quantified arguments containing predicates feature in the same syntactic roles as names in Quarc, it has two different sets of existence assumptions - those of non-emptiness of names, and also of predicates. As noted in the introduction, we will be dropping both of those in this paper.

In this section, we restate the axioms to talk not of individuals, referred to by constants (or singular arguments in the terminology of Quarc), but of "some M's", captured by unary predicates. In what [15] refer to as full Quarc, these are required to be non-empty, but both systems under consideration here, LKQuarc $_{B}$ and LK-Quarc 2 , omit that requirement. 
It should be noted that restricted specification as applied to predicates instead of names, $\forall M A \rightarrow \exists M A$, is not valid in either of those systems [16], and therefore neither is it so in $\mathrm{FQ}_{B}$ (by Corollary 6), nor in $\mathrm{FQ}_{2}$ (by Theorem 13). This checks off the first requirement on being able to describe a system as a (negative) free logic. As importantly, all the axioms must likewise hold, and this is in fact the case, restated for "some M's":

Theorem 17 All of the following axioms are derivable in $\mathrm{FQ}_{B}\left[\mathrm{FQ}_{2}\right]$ :

1. $\left(\forall M_{\alpha} A \rightarrow \alpha B\right) \rightarrow(\forall M A \rightarrow \forall M B)$

2. $A \rightarrow(\forall M M \wedge B)$

3. $\forall M A \rightarrow(\exists M E ! \rightarrow \exists M A)$

4. $\forall M E$ !

5. $\exists M P \rightarrow \exists M E$ !

6. $\left[\forall M_{\alpha}=\alpha\right.$, for any $\left.M\right]$

7. $[\exists M=\exists P \rightarrow(\exists M A \rightarrow \exists P A)]$

So, both $\mathrm{FQ}_{B}$ and $\mathrm{FQ}_{2}$ are free not just with respect to non-empty names, but also non-empty predicates. That this feature transfers back to LK-Quarc ${ }_{B}$ and LK-Quarc 2 can be demonstrated by restating the axioms without the existence predicate $E$ !. We are able to do this, when talking about some M's, since (given the close connection between unary predicates and existence predicate), for some $M$ to exist, $E ! M$, is for it to be some unary predicate, namely $M, \exists M M$ :

Lemma $18 \exists M E ! \leftrightarrow \exists M M$

The left-to-right direction is obtained by a simple use of $\mathrm{R} \exists$ and then $\mathrm{L} \exists$, and the right-to-left direction is an instance of the Axiom 5 from the Theorem 17.

It follows from Theorem 17 and Lemma 18, again using Corollary 6 and Theorem 13 that

Theorem 19 All of the following axioms are derivable in LK-Quarc ${ }_{B}$ [LKQuarc $_{2}$ ]:

1. $\left(\forall M_{\alpha} A \rightarrow \alpha B\right) \rightarrow(\forall M A \rightarrow \forall M B)$

2. $A \rightarrow(\forall M M \wedge B)$

3. $\forall M A \rightarrow(\exists M M \rightarrow \exists M A)$

4. $\left(\forall M_{\beta} M \wedge\left(\forall M_{\alpha} M \wedge A[\alpha, \beta]\right)\right) \rightarrow\left(\forall M_{\alpha} M \wedge\left(\forall M_{\beta} M \wedge A[\alpha, \beta]\right)\right)[\forall M=\exists M]$

5. $\exists M P \rightarrow \exists M M$

6. $\left[\forall M_{\alpha}=\alpha\right.$, for any $\left.M\right]$

7. $[\exists M=\exists P \rightarrow(\exists M A \rightarrow \exists P A)]$ 
A note on Axiom 4 - for LK-Quarc 2 , a very elegant axiom is available (as elsewhere, written within square brackets). However, since it requires the identity predicate, we cannot use it for LK-Quarc ${ }_{B}$. Instead, what we do here is emulate the Permutation Principle [6] in Quarc.

We can now strengthen the Observation 16 and conclude that

Observation 20 The quantification rules of Quarc, even on the non-free version and with respect to both emptiness of names, as well as that of unary predicates, bear a structural resemblance to those of free logic, specifically negative free logic.

\section{References}

[1] Baaz, Matthias/Leitsch, Alexander (2011): Methods of Cut-Elimination. Dordrecht: Springer.

[2] Bencivenga, Ermano (2002): "Free Logics". In: Handbook of Philosophical Logic. 2nd Edition, Vol. 5. Dov Gabbay, Franz Guenthner (Eds.). Dordrecht: Springer, pp. 147-196.

[3] Ben-Yami, Hanoch (2004): Logic and Natural Language: on plural reference and its semantic and logical significance. Aldershot: Ashgate.

[4] Ben-Yami, Hanoch (2014): "The Quantified Argument Calculus". In: The Review of Symbolic Logic 7 No. 1, pp. 120-146.

[5] Buss, Samuel (1998): "An Introduction to Proof Theory". In: Handbook of Proof Theory. Vol. 137. Samuel Buss (Ed.). Amsterdam: Elsevier, pp.1-78.

[6] Fine, Kit (1983): "The Permutation Principle in Quantificational Logic". In: Journal of Philosophical Logic 12 No. 1, pp. 33-37.

[7] Gentzen, Gerhard (1969): The Collected Papers of Gerhard Gentzen. M.E. Szabo (Ed.). Amsterdam: North-Holland Pub. Co..

[8] Gratzl, Norbert (2010): "A Sequent Calculus for a Negative Free Logic". In: Studia Logica 96, pp. 331-348.

[9] Kleene, Stephen Cole (2000): Introduction to Metamathematics. Amsterdam: Elsevier.

[10] Lambert, Karel (1997): Free Logics: Their Foundations, Character, and Some Applications Thereof. Sankt Augustin: Academia Verlag.

[11] Lambert, Karel (2001): "Free Logics". In: The Blackwell Guide to Philosophical Logic. Lou Goble (Ed.). Oxford: Blackwell Publishers, pp.258-279.

[12] Lanzet, Ran/Ben-Yami, Hanoch (2006): "Logical Inquiries into a New Formal System with Plural Reference". In: First Order Logic Revisited. Vincent F. Hendricks (Ed.). Berlin: Logos Verlag, pp. 173-223. 
[13] Lanzet, Ran (2017): "A Three-Valued Quantified Argument Calculus: domain-free model theory, completeness, and embedding of FOL". In: The Review of Symbolic Logic 10, No. 3, pp. 549-582.

[14] Negri, Sara/von Plato, Jan (2001): Structural Proof Theory. Cambridge: Cambridge University Press.

[15] Pavlović, Edi/Gratzl, Norbert (Forthcoming 2019): "Proof-Theoretic Analysis of the Quantified Argument Calculus". In: The Review of Symbolic Logic.

[16] Pavlović, Edi (2017): The Quantified Argument Calculus: an inquiry into its logical properties and applications. PhD Thesis, Central European University, Budapest.

[17] Schütte, Kurt (1960): Beweistheorie. Berlin-Göttingen-Heidelberg: Springer.

[18] Takeuti, Gaisi (1987): Proof Theory. 2nd Edition. Amsterdam-LausanneNew York-Oxford-Tokyo: North Holland. 